\title{
Finite elements for problems of the elasticity theory with the discontinuous stress approximation
}

\author{
$A$ Lukashevich $^{1,}, N$ Lukashevich $^{1}$, and $E$ Kobelev $^{1}$ \\ ${ }^{1}$ Saint Petersburg State University of Architecture and Civil Engineering, 4, Vtoraya \\ Kranoarmeiskaya, 190005, Saint Petersburg, Russia
}

\begin{abstract}
The paper deals with the development of the finite element models on the basis of stress approximation. At present, the displacementbased finite element method is mainly used for engineering calculations. Finite element formulations in stresses are not so widely spread, but in some cases these formulations can be more effective in particular with respect to the calculating stresses and also obtaining a two-sided estimate of the exact solution of the problem. The finite element models based on the approximation of discontinuous stress fields and the use of the penalty function method to satisfy the equilibrium equations are considered. It is shown that the continuity of both normal and tangential stresses only on the adjacent sides of the finite elements contributes to the expansion of the class of statically admissible stress fields. At the same time, the consistent approximation is provided, both of the main part of the functional of additional energy, and its penalty part. The necessary matrix relations for rectangular and triangular finite elements are obtained. The effectiveness of the developed models is illustrated by numerical studies. The calculation results were compared with the solution on the FEM in displacements, as well as with the results obtained using other schemes of approximating the stresses in the finite element. It is shown that the model of discontinuous stress approximations gives the bottom convergence of the solution, both in stresses and in displacements. At the same time, the accuracy on the stresses here is much higher than in the displacement-based FEM or when using conventional stress approximation schemes.
\end{abstract}

\section{Introduction}

Nowadays, the calculation of structure is carried out, as a rule, using the finite element method (FEM). Many fundamental works have been devoted to the development of the theoretical principles of the FEM and questions of its application in structural mechanics [1, 2]. They consider the basic variational principles and the corresponding FEM formulations, on the basis of which the finite element models can be constructed to solve different problems.

\footnotetext{
*Corresponding author: aaluk@bk.ru
} 
There are three main forms of FEM, each form is an analogue of one of the three classical methods of structural mechanics of rod systems - the displacement method, the force method, and the mixed method. The most widely used engineering calculations method is the FEM in the displacement method form. The mathematical formulation of the problem here is based on the variational Lagrange principle, i.e., the minimum principle of the total potential energy of the system. The main unknowns in this case are the displacements of the nodal points of the discrete model of the structure. The stresses are secondary and can be calculated by numerically differentiating the displacements. The finite element method in displacements is widely used to solve geometrically and physically nonlinear problems [3-5], constructively nonlinear problems with unilateral constraints [6-9], problems of stability and dynamics of structures [10-13].

The advantages and disadvantages of the displacement method are well known. The huge advantage of this FEM form is its complete formalization (and, accordingly, the ease of implementation to software), as well as good stability of the solution with guaranteed convergence to the lower boundary. However, the accuracy of stresses (forces) determination is much lower than the displacements, although it is stress values which are more important in the structural strength analysis. In addition, since the approximate solution in displacements corresponds to the lower boundary, the values of both displacements and stresses are underestimated relative to the exact values. Attempts to overcome these shortcomings, based on the use of FEM schemes directly in stresses (forces) or in a mixed form, have been made repeatedly, but this problem is still far from complete and remains one of the most important problems of the finite element method application in structural mechanics.

Castiliano's minimum principle of additional energy and the related FEM schemes in the form of the force method, as well as the Reissner variational principle (mixed method), have not received such a wide application. This situation is caused by a number of circumstances, in particular, the need to satisfy the equilibrium equations in the force method or an increase in the number of unknowns in the mixed method. However, in some cases, these approaches can be more effective, especially with regard to calculated stresses. In addition, performing dual calculations based on the alternative forms of FEM allows, as a rule, to obtain a twoside estimate of the exact solution of the problem [1].

The FEM variants, in which the search of the solution is based on the approximation of stresses (forces) in the finite element region, are considered in [1, 14-20]. In [17], the combination of principles of possible displacements and possible changes in the stress state is used to find the solution. The solution to the problems of the elasticity theory in stresses on the basis of the functional of additional energy is considered in $[19,20]$. In this case, the principle of possible displacements and Lagrange multipliers are used to perform the equilibrium equations.

The main advantage of the FEM in the form of the force method is that the main unknowns here are stresses. And if there were no certain difficulties in implementing the force method [2], the stress values could be obtained with the same degree of accuracy as the displacements in the FEM scheme of the same name. In addition, the use of Castiliano's principle gives the upper boundary of the approximate solution (i.e., the stresses are overestimated), which, in principle, is better for strength calculations than the underestimated estimate. On the other hand, solving the problem in stresses can supplement the usual calculation of FEM in displacements, in particular, from the point of view of the two-sided estimation of the solution of the problem on energy. Nevertheless, there are no algorithms that are equally as simple and stable, with guaranteed convergence in an extensive class of problems, similar to the FEM in the form of the displacement method.

This paper is devoted to the development of FEM for solving problems of the elasticity theory in stresses. It is proposed to use the approximation of discontinuous stress fields in the 
finite element region to construct a solution to the plane elasticity problem. The proposed finite element model may have advantages in terms of more accurately fulfilling the equilibrium conditions. At the same time, the accuracy of stress determination here is much higher than in the displacement-based FEM or when using conventional stress approximation schemes.

\section{Methods}

Let us consider the following version of the FEM in the force method form, which allows us to solve a wide class of problems directly in stresses efficiently. It is based on the approximation of discontinuous stress fields and the use of the penalty method to satisfy equilibrium equations. The corresponding variational formulation for the plane problem of the elasticity theory has the form of a modified functional of additional energy:

$$
\begin{aligned}
\Pi(\sigma)= & -\frac{1}{2} \int_{\Omega}\{\sigma\}^{T}[D]^{-1}\{\sigma\} d \Omega+\int_{S_{u}}\left\{u_{s}\right\}^{T}\left[L_{s}\right]^{T}\{\sigma\} d S+ \\
& +\alpha \int_{\Omega}\left([A]^{T}\{\sigma\}+\{\rho\}\right)^{T}\left([A]^{T}\{\sigma\}+\{\rho\}\right) d \Omega
\end{aligned}
$$

under the additional condition at the region boundary: $\left[L_{s}\right]^{T}\{\sigma\}=\left\{g_{s}\right\} \in S_{g}$.

Here $\boldsymbol{\sigma}=\left\{\begin{array}{lll}\sigma_{x} & \sigma_{y} & \tau_{x y}\end{array}\right\}^{T}$ is the stress vector; $[D]$ is the stiffness coefficients matrix; $[A]$ is the differentiation operations matrix in the equilibrium equations; ${ }^{\left[L_{s}\right]}$ is the direction cosine matrix of the external normal to the boundary $S=S_{g}+S_{u}$ of the plane region $\Omega ;\left\{g_{s}\right\}$ is the surface force vector at the boundary $S_{g} ;\left\{u_{s}\right\}$ is the vector of given displacements at the $S_{u} ;\{\rho\}=\left\{\rho_{x} \rho_{y}\right\}^{T}$ is the volumetric force vector.

When solving problems in stresses, the main restrictions on the smoothness of the desired functions are imposed by the equilibrium equations - it is necessary to ensure the existence of piecewise continuous derivatives of the stress components. Therefore, according to them, for the plane problem of the elasticity theory, the differentiability of the stresses $\sigma_{x}$ only on $x,^{\sigma_{y}}$ - only on $y$, and $\tau_{x y}$ - both on $x$ and on $y$ is required. Thus, normal stresses can undergo discontinuities at sites perpendicular to the element boundaries. In comparison with the continuous approximation, the use of such an assumption contributes to the expansion of the class of statically allowable stress fields, among which the solution is sought. This allows to minimize the additional energy functional to a greater extent - as a result, the solution of the problem is, on average, closer to the exact one. The use of discontinuous approximations leads to the need to apply a special class of finite elements. The location of the nodal points here should ensure that the conditions of discontinuity of normal stresses are satisfied.

Figure 1 shows one of the simplest elements of this kind, which is a rectangle, the four nodes at the vertices of which correspond to tangential stresses, two nodes on each of the sides correspond to normal stresses $\sigma_{x}$, two nodes on the upper and lower sides correspond to stresses $\sigma_{y}$. 


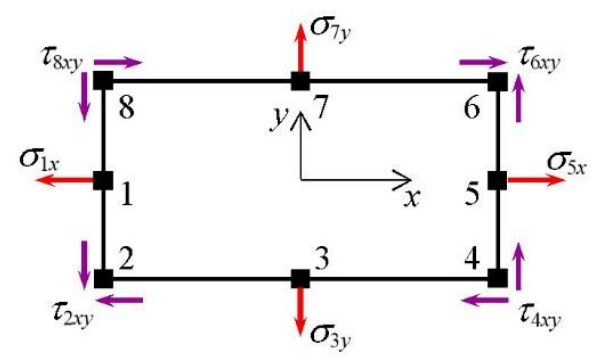

Fig. 1. Eight-node finite element with discontinuous stress approximation.

Here the stress distribution is given by the following approximating polynomials:

$$
\sigma_{x}=\alpha_{1}+\alpha_{2} x ; \quad \sigma_{y}=\alpha_{3}+\alpha_{4} y ; \quad \tau_{x y}=\alpha_{5}+\alpha_{6} x+\alpha_{7} y+\alpha_{8} x y .
$$

Having substituted the approximation data in the equilibrium equations, we obtain:

$$
\alpha_{2}+\alpha_{7}+\alpha_{8} x+\rho_{x}=0 ; \alpha_{4}+\alpha_{6}+\alpha_{8} y+\rho_{y}=0 .
$$

Obviously, for the constant volumetric element forces $\rho_{x}, \rho_{y}$, the conditions (3) can be satisfied only if the following condition is identically satisfied: $\alpha_{8} x=\alpha_{8} y=0$.

Since this is not provided by the exact integration of the penalty term of functional in Eq. (1), a method for artificial lowering the accuracy of calculating the integrals was proposed for the reduced finite element, which facilitates the zeroing of the expression in the integral part of the penalty term [7]. Despite the fact that this finite element gives the acceptable accuracy and convergence of the results of solving problems in stresses, there are significant disadvantages. First, to calculate the coefficients of the deformability matrix related to the penalty term, it is necessary to apply the procedure of numerical integration. Secondly, for the main part of functional (1) and its penalty term, different stress approximations are used - for the penalty term, they are respectively of a lower order than for the main part of the functional. The model proposed here for approximating discontinuous stress fields for a finite element of a plane problem of the elasticity theory is free from these disadvantages.

Consider the above condition $\alpha_{8} x=\alpha_{8} y=0$. For the finite element (figure 1) and approximation (2) described above, its exact execution is possible only when using the singlepoint integration scheme of the penalty term, i.e. when the integration order is lowered. However, locating nodes of the finite element on the $x, y$ axes (figure $2 \mathrm{a}$ ), the condition $\alpha_{8} x=\alpha_{8} y=0$ is reduced to the equality $\alpha_{8} x=\alpha_{8} y$.

(a)

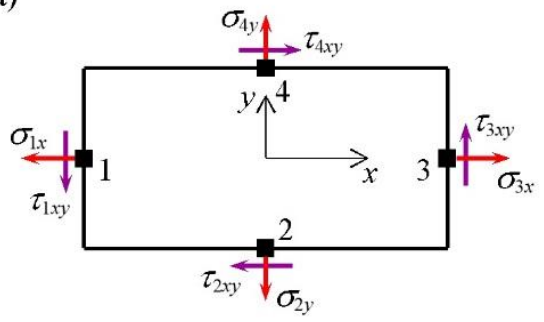

(b)

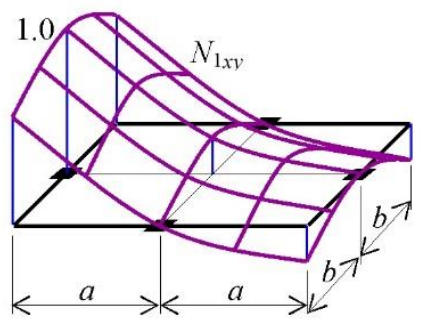


Fig. 2. Four-node finite element with discontinuous stress approximation: (a) location of the nodes; (b) approximation of the tangential stresses.

Substituting this equality into approximation (2), we get the following polynomial variants for $\tau_{x y}$ :

$$
\tau_{x y}=\alpha_{5}+\alpha_{6} x+\alpha_{7} y+\alpha_{8} x^{2} ; \quad \tau_{x y}=\alpha_{5}+\alpha_{6} x+\alpha_{7} y+\alpha_{8} y^{2} .
$$

For each of the polynomials (4), we define the form functions for nodes and average them. Then the nodal stress vector for the finite element $\{\sigma\}^{e}=\left\{\begin{array}{llllllll}\sigma_{1 x} & \tau_{1 x y} & \sigma_{2 y} & \tau_{2 x y} & \sigma_{3 x} & \tau_{3 x y} & \sigma_{4 y} & \tau_{4 x y}\end{array}\right\}^{T}$ the following form functions will be a respond:

$$
N_{1,3 x}=\frac{1}{2} \mp \frac{x}{2 a} ; \quad N_{1,3 x y}=\frac{1}{4} \mp \frac{x}{2 a}+\frac{x^{2}}{4 a^{2}}-\frac{y^{2}}{4 b^{2}}, \quad N_{2,4 y}=\frac{1}{2} \mp \frac{y}{2 b} ; \quad N_{2,4 x y}=\frac{1}{4} \mp \frac{y}{2 b}-\frac{x^{2}}{4 a^{2}}+\frac{y^{2}}{4 b^{2}} \text {. }
$$

The approximation of the tangential stresses within a given finite element, in particular, for the shape function $N_{1 x y}$, is shown in figure $2 \mathrm{~b}$.

Expressing the stresses using the form functions through the nodal values and substituting in the stationarity condition of the functional (1) for finite element, we obtain the following matrix equation:

$$
\left[K_{\sigma}\right]^{e}\{\sigma\}^{e}=\{U\}^{e}
$$

The deformability matrix of the finite element can be represented as the sum of two matrixes

$$
\begin{aligned}
& {\left[K_{\sigma}\right]^{e}=\left[K_{0}\right]^{e}+\left[K_{\alpha}\right]^{e} \text {. The first term }\left[K_{0}\right]^{e}=\int_{\Omega}\left[N_{\sigma}\right]^{T}[D]^{-1}\left[N_{\sigma}\right] d \Omega} \\
& \qquad\left[K_{\alpha}\right]^{e}=-\alpha \int_{\Omega}\left([A]^{T}\left[N_{\sigma}\right]\right)^{T}[A]^{T}\left[N_{\sigma}\right] d \Omega \\
& \text { the main part of the functional (1), the second to }
\end{aligned}
$$

its penalty part. The right part is the vector of given nodal displacements $\{U\}^{e}=\int_{S_{u}}\left[N_{\sigma}\right]^{T}\left[L_{s}\right]^{-1}\left\{u_{s}\right\} d S+\alpha \int_{\Omega}\left([A]^{T}\left[N_{\sigma}\right]\right)^{T}\{\rho\} d \Omega$

$\left[N_{\sigma}\right]=\left[\begin{array}{cccccccc}N_{1 x} & 0 & 0 & 0 & N_{3 x} & 0 & 0 & 0 \\ 0 & 0 & N_{2 y} & 0 & 0 & 0 & N_{4 y} & 0 \\ 0 & N_{1 x y} & 0 & N_{2 x y} & 0 & N_{3 x y} & 0 & N_{4 x y}\end{array}\right]$ is the matrix of form functions for stresses in finite element.

Having done the matrix operations and integration the obtained expressions, we obtain: 


$$
\begin{aligned}
& {\left[K_{0}\right]^{e}=\kappa\left[\begin{array}{cccccccc}
113 \mu & 0 & 37 \mu & 0 & -7 \mu & 0 & 37 \mu & 0 \\
0 & 240 & 0 & -180 v & 0 & 120 & 0 & -180 v \\
37 \mu & 0 & 113 \mu & 0 & 37 \mu & 0 & -7 \mu & 0 \\
0 & -180 v & 0 & 240 & 0 & -180 v & 0 & 120 \\
-7 \mu & 0 & 37 \mu & 0 & 113 \mu & 0 & 37 \mu & 0 \\
0 & 120 & 0 & -180 v & 0 & 240 & 0 & -180 v \\
37 \mu & 0 & -7 \mu & 0 & 37 \mu & 0 & 113 \mu & 0 \\
0 & -180 v & 0 & 120 & 0 & -180 v & 0 & 240
\end{array}\right]} \\
& {\left[K_{\alpha}\right]^{e}=\alpha\left[\begin{array}{cccccccc}
4 m+n & 0 & -m-n & -1 & n-2 m & 0 & -m-n & 1 \\
0 & 3 m & -1 & 0 & 0 & -3 m & 1 & 0 \\
-m-n & -1 & m+4 n & 0 & -m-n & 1 & m-2 n & 0 \\
-1 & 0 & 0 & 3 n & 1 & 0 & 0 & -3 n \\
n-2 m & 0 & -m-n & 1 & 4 m+n & 0 & -m-n & -1 \\
0 & -3 m & 1 & 0 & 0 & 3 m & -1 & 0 \\
-m-n & 1 & m-2 n & 0 & -m-n & -1 & m+4 n & 0 \\
1 & 0 & 0 & -3 n & -1 & 0 & 0 & 3 n
\end{array}\right] .}
\end{aligned}
$$
and Poisson's ratio; $a, b$ are the size of the rectangular finite element.

The penalty parameter $\alpha$ has the physical meaning of the deformability coefficient of the weakest possible elastic base. Its value should be large enough and limited from above only by the accuracy of calculations. The recommendations for choosing the values $\alpha$ for different problems are given in [19]. As applied to the plane problem of the elasticity theory, $\alpha=\left(10^{7} \div 10^{9}\right) \kappa$ can be accepted.

Similarly, the approximation of discontinuous stress fields can be applied to construct finite elements in stresses of other geometric shapes, as well as finite elements of the mixed form. For example, for a finite element in the form of a right triangle (figure $3 \mathrm{a}$ ), the stress distribution can be represented by the following polynomials:

$$
\sigma_{x}=\alpha_{1}+\alpha_{2} x ; \quad \sigma_{y}=\alpha_{3}+\alpha_{4} y ; \quad \tau_{x y}=\alpha_{5}+\alpha_{6} x+\alpha_{7} y
$$

It is clear that the fulfillment of the condition similar to $\alpha_{8} x=\alpha_{8} y=0$ is not required here. The following form functions will respond to the vector of nodal stresses of a finite element

$$
\begin{aligned}
\{\sigma\}^{e}= & \left\{\begin{array}{lllllll}
\sigma_{1 x} & \tau_{1 x y} & \sigma_{2 y} & \tau_{2 x y} & \sigma_{3 x} & \sigma_{3 y} & \tau_{3 x y}
\end{array}\right\}^{T}: \\
& N_{1 x}=-\frac{x}{a} ; \quad N_{1 x y}=-\frac{x}{a} ; \quad N_{2 y}=-\frac{y}{b} ; \quad N_{2 x y}=-\frac{y}{b} ; \quad N_{3 x}=1+\frac{x}{a} \quad N_{3 y}=1+\frac{y}{b} ; \quad N_{3 x y}=1+\frac{x}{a}+\frac{y}{b} .
\end{aligned}
$$


We replace the nodal stress components $\sigma_{3 y}, \sigma_{3 x}, \tau_{3 x y}$ with the tangent and normal components to the inclined face of the element $\sigma_{3 n}, \tau_{3 n}$ (figure $3 \mathrm{~b}$ ). The nodal stress vector will then be $\{\sigma\}^{e}=\left\{\sigma_{1 x} \tau_{1 x y} \sigma_{2 y} \tau_{2 x y} \sigma_{3 n} \tau_{3 n}\right\}^{T}$.

(a)

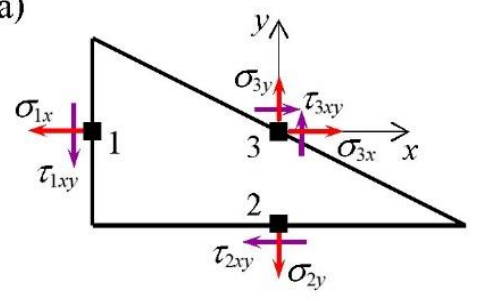

(b)

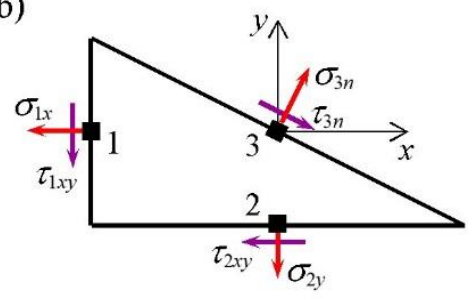

Fig. 3. Triangular finite element with discontinuous stress approximation.

After substituting the form functions and converting the stress components during turn of the axes, we obtain the following terms of the deformability matrix of a triangular finite element:

$$
\begin{aligned}
& {\left[K_{0}\right]^{e}=\kappa\left[\begin{array}{cccccc}
\lambda & 0 & 0 & 0 & 0 & 0 \\
0 & 1 & v l m & -v l^{2} & 0 & 0 \\
0 & v l m & \lambda\left(l^{2}-m^{2}\right) & \operatorname{lm}(2 \lambda+2+v) & 0 & -\operatorname{lm}(2+v) \\
0 & -v l^{2} & \operatorname{lm}(2 \lambda+2+v) & 2\left(m^{2}-v l^{2}\right) & 0 & -v m^{2} \\
0 & 0 & 0 & 0 & \lambda & 0 \\
0 & 0 & -\operatorname{lm}(2+v) & -v m^{2} & 0 & 1
\end{array}\right]} \\
& {\left[K_{\alpha}\right]^{e}=\alpha\left[\begin{array}{cccccc}
\eta & 0 & l m-\eta \chi & -l^{2}-2 \eta \cdot l m & 1 & -1 \\
0 & \eta & -\eta \cdot l m-\chi & -2 l m-\eta \cdot m^{2} & 0 & 0 \\
l m-\eta \chi & -\eta \cdot l m-\chi & \chi \cdot \beta & \chi+\eta \cdot l m+\omega+2 \beta \cdot l m & -l m-\mu \chi & \mu \cdot l m-\chi \\
-l^{2}-2 \eta \cdot l m & -2 l m-\eta \cdot m^{2} & \chi+\eta \cdot l m+\omega+2 \beta \cdot l m & \eta \cdot m^{2}+4 l m & -m^{2}-2 \mu \cdot l m & -2 l m-\mu \cdot l^{2} \\
1 & 0 & -l m-\mu \chi & -m^{2}-2 \mu \cdot l m & \mu & -\mu \\
-1 & 0 & \mu \cdot l m-\chi & -2 l m-\mu \cdot l^{2} & -\mu & \mu
\end{array}\right] .} \\
& \text { Here: } \quad \kappa=\frac{2 a b}{3 E} ; \lambda=2(1+v) ; \eta=\frac{a}{b} ; \mu=\frac{b}{a} ; \beta=\eta+\mu ; \chi=\left(l^{2}-m^{2}\right) \text {, where } E, v
\end{aligned}
$$

\section{Results and discussion}


Let us consider the problem of bending a cantilever plate under the action of a vertical distributed load applied to its free end is presented below in figure $4 \mathrm{a}$. The plate thickness is $1 \mathrm{~m}$, the elastic modulus is $10000 \mathrm{kN} / \mathrm{m}^{2}$, the Poisson's ratio is 0.25 . This example was given in [19], where the application of the finite element models in stresses at constant and piecewise-constant approximations of stresses in the finite element region was considered. Below, we compare the solutions constructed on the basis of approximations of discontinuous stress fields with the results obtained in [19] using other schemes of stress approximation in the finite element.

Table 1 shows the stress values in the upper fibers of the section at the clamp $(x=0, y=$ $1 \mathrm{~m}$ ), obtained using different stress approximation schemes in the finite element. The calculation results are presented for four variants of the finite element grids. For comparison, the lower row of the table shows the stress values obtained by FEM in the LIRA-SAPR software package for a relatively dense grid $192 \times 64$.

Table 1. Comparison of the beam calculation results for different types of stress approximation.

\begin{tabular}{|c|c|c|c|c|c|c|c|c|c|}
\hline \multirow{2}{*}{ Grid } & \multicolumn{3}{|c|}{ Constant stresses } & \multicolumn{3}{|c|}{$\begin{array}{c}\text { Piecewise constant } \\
\text { stresses }\end{array}$} & \multicolumn{3}{|c|}{ Discontinuous stresses } \\
\hline & $\begin{array}{c}\sigma_{x} \\
\mathbf{k N} / \mathbf{m}^{2}\end{array}$ & $\begin{array}{c}\sigma_{y}, \\
k N / m^{2}\end{array}$ & $\begin{array}{c}\tau_{x y}, \\
\mathrm{kN} / \mathrm{m}^{2}\end{array}$ & $\begin{array}{c}\sigma_{x}, \\
\mathbf{k N} / \mathbf{m}^{2}\end{array}$ & $\begin{array}{c}\sigma_{y}, \\
\mathbf{k N} / \mathbf{m}^{2}\end{array}$ & $\begin{array}{c}\tau_{x y}, \\
\mathbf{k N} / \mathbf{m}^{2}\end{array}$ & $\begin{array}{c}\sigma_{x} \\
\mathbf{k N} / \mathbf{m}^{2}\end{array}$ & $\begin{array}{c}\sigma_{y}, \\
\mathbf{k N} / \mathbf{m}^{2}\end{array}$ & $\begin{array}{c}\tau_{x y} \\
\mathrm{kN} / \mathbf{m}^{2}\end{array}$ \\
\hline $12 \times 4$ & 13.96 & 0.95 & 1.11 & 17.56 & 3.12 & 1.75 & 22.18 & 3.42 & 2.75 \\
\hline $24 \times 8$ & 16.53 & 1.43 & 1.45 & 19.55 & 3.52 & 2.40 & 23.25 & 3.67 & 3.08 \\
\hline $48 \times 16$ & 18.91 & 1.78 & 1.93 & 22.06 & 4.03 & 3.22 & 23.62 & 3.80 & 3.44 \\
\hline $96 \times 32$ & 21.45 & 2.10 & 2.49 & 25.16 & 4.65 & 4.06 & 24.17 & 3.97 & 3.69 \\
\hline $192 \times 64$ & 24.02 & 3.46 & 3.62 & 24.02 & 3.46 & 3.62 & 24.02 & 3.46 & 3.62 \\
\hline
\end{tabular}

The table shows that even on the coarse grids, the stress values obtained on the basis of discontinuous approximations are more accurate compared to using the constant and piecewise constant stress approximations. So, for the grid $12 \times 4$, the stress values $\sigma_{x}$ differ upward from the constant and piecewise-constant stresses by $37 \%$ and $21 \%$, respectively.

The stresses $\sigma_{y}$ and $\tau_{x y}$ in our case are also much more accurate than with ordinary schemes of approximations.

When the grid is crushed, the model of discontinuous approximations gives a monotonous and stable convergence of stresses to the lower boundary. In this case, the numerical stress values are in the gap between the stress values obtained with the constant and piecewiseconstant approximations. For example, for grid $96 \times 32$, the stress $\sigma_{x}$ is $11 \%$ higher than at constant stresses in the element, but $4 \%$ less than for the piecewise-constant stresses variant. Figures $4 \mathrm{~b}-\mathrm{d}$ illustrate the convergence of solutions in stresses for different types of stress approximations. 
(a)

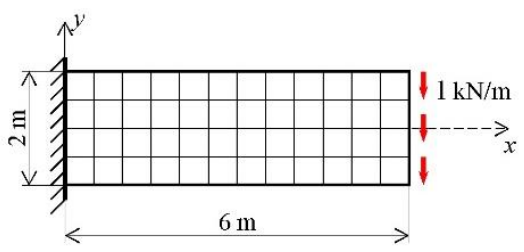

(c)

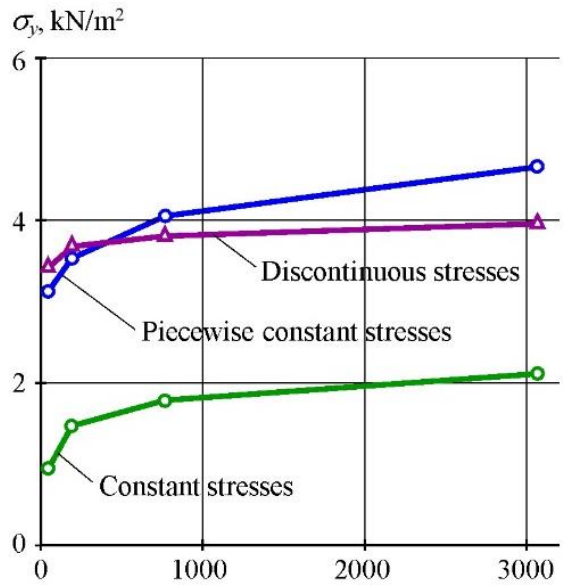

(b)

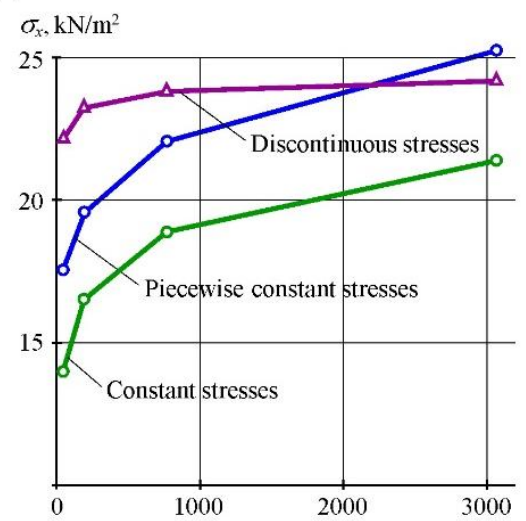

(d)

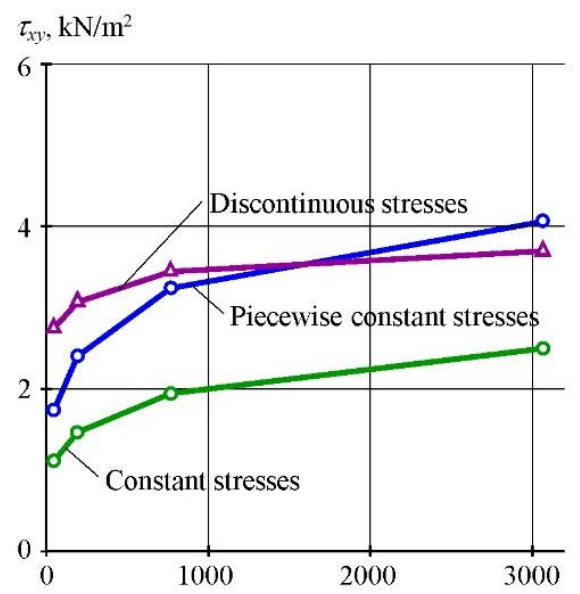

Fig. 4. The convergence of solutions in stresses for different types of approximation.

\section{Conclusions}

Summarizing the results of numerical researches, we can draw the following conclusions:

1. The finite element method in the form of the force method in the general case gives the upper convergence of the solution in stresses. This allows us to obtain a two-sided estimate of the exact solution of the corresponding problem. At the same time, the finite-element solutions built on the stress approximation are much closer to exact values than those obtained in the displacement method formulation.

2. The use of discontinuous stress approximations contributes to the expansion of the class of statically admissible stress fields. In this case, the same approximation is provided for both the main part of the additional energy functional and its penalty part. This allows us to obtain more accurate stress values as compared to the continuous stress approximation.

3. The finite element models in stresses allow to obtain alternative solutions to the considered problems, thereby ensuring their greater validity and reliability.

4. Discontinuous stress approximations give a fast and stable convergence of the resulting solution to the lower boundary. At the same time, the accuracy of the stress in this case is 
significantly higher than when using conventional stress approximation schemes, in particular, constant and piecewise constant approximations.

\section{References}

1. Zienkiewicz O C, Taylor R L and Zhu J Z 2013 The Finite Element Method: Its Basis and Fundamentals (Butterworth-Heinemann) p 756

2. Bathe K J 2016 Finite element procedures (Englewood Cliffs: Prentice Hall) p 1043

3. Lalin V V, Rybakov V A and Morozov S A 2012 Magazine of Civil Engineering 27 53-73

4. Paola M and Scimemi G 2016 Computers \& Structures 164 15-22

5. Lukashevich A A and Lukashevich N K 2019 IOP Conf. Ser.: Mater. Sci. Eng. 687 033024

6. Wriggers P, Schroder J and Schwarz A 2013 Computational Mechanics 52 837-847

7. Lukashevich A A 2014 Advanced materials research 941 2264-2267

8. Wriggers P, Rust W T and Reddy B D 2016 Computational Mechanics 58 1039-1050

9. Lukashevich A A 2018 Magazine of civil engineering 81 149-159

10. Rybakov V A, Lalin V V, Ivanov S S and Azarov A A 2019 Magazine of Civil Engineering 89 115-128

11. Lukashevich A A 2019 Magazine of civil engineering 89 167-178

12. Alshurafa S, Hanan H and Polyzois D 2019 Journal of Computational Design and Engineering 6(3) 436-446

13. Lukashevich A A and Lukashevich N K 2020 IOP Conf. Ser.: Mater. Sci. Eng. $\mathbf{7 5 3}$ 022058

14. Muller B and Starke G 2016 Advanced Finite Element Technologies 69-104

15. Kuss F and Lebon F 2009 Advances in Engineering Software 40(8) 697-706

16. Kuo Y-L 2015 Appl. Math. Inf. Sci. 9(2) 609-616

17. Negrozov O A and Akimov P A 2017 AIP Conference Proceedings 1800040014

18. Peng Y, Bai Y, Guo Q 2017 Structural Engineering and Mechanics 62 11-20

19. Tyukalov Y Y 2018 Magazine of Civil Engineering 77 23-37

20. Tyukalov Y Y 2019 Magazine of Civil Engineering 91 80-97 